\title{
Regenerative Dentistry
}

\author{
Paul Sharpe* \\ Centre for Craniofacial and Regenerative Biology, Faculty of Dentistry, Oral \& Craniofacial Sciences, Kings College London, \\ London, United Kingdom
}

Keywords: challenges, stem cells, tissue repair, clinical translation, dental

What do we mean by the term "regenerative" dentistry? In my view it simply means using an understanding of cell and molecular biology to design dental therapies that aim to restore, repair, rejuvenate, and regenerate dental tissues. The issue of which of these, often misused terms, is used, is not important. What is important is an appreciation of the overall concept and its massive implications for the future of clinical dentistry.

Few, if any current dental treatments are based on tissue biology or directed toward tissue regeneration. In medicine however, almost all treatments are based on an understanding of the biology of the tissue or disease processes and the latest developments in gene editing, genomics, single cell analysis, and stem cells are rapidly driving medicine toward a new era. I see no reason why dentistry should not embrace these advances and begin a major drive to move beyond the from the continuing introduction of yet more non-biological materials-based incremental "improvements" in treatments. The Grand Challenge for Regenerative Dentistry is to experdite this drive through high quality research, clinical translation, and education to enable a new era of dental treatments, based on an understanding of natural dental biological processes, to rapidly enter the clinic.

Teeth and their support tissues provide an easily accessible "treasure chest" of stem cell populations. Tooth pulp, growing roots, periodontal ligament, and alveolar bone are sources that has been exploited as a source of generic stem cells with properties in vitro similar to those original described for bone marrow stromal (mesenchymal) stem cells. The advent of in vivo genetic lineage tracing techniques in mice, is now questioning the extent to which such adult cells maintained in in vitro culture can be considered stem cells, namely self-renewing cells capable of multilineage differentiation, rather than lineage restricted, non-self-renewing progenitors. Single cell transcriptomics are revealing the heterogeneity of stem cell populations, including multiple cell origins and differentiation potentials. The ability (or lack of) of cultured adult "stem" cells to contribute to cell differentiation following transplantation remains a major issue in tissue engineering and the development of even smarter bioactive delivery vehicles based on hydrogels can only lead to improvements in transplanted cell viability and function. The more we learn about stem cells, their niches, their preprogramming, and their transitions in vivo the more it is time to move on from the concept of generic, one suits all, "stem cells in a dish" for tissue engineering applications.

The basic molecular processes that control tooth development are very well-understood thanks to $20+$ years of intensive research. Such understanding provides the necessary background for adult tissue regeneration starting with the holy grail, the formation of a complete biological replacement tooth. Remarkedly, such teeth can be easily produced by transplantation of artificially-constructed embryonic tooth primordia into the adult oral cavity. Since 2004 it has been established that tooth primordia can be made when one of the two embryonic cell populations replaced by adult cells and that such primordia will continue to develop into functional teeth following transplantation. Thus, the principle of a bioengineered tooth explant as a replacement for a metal dental implant has been established. What remains is to generate such "Bioteeth" entirely from adult cells, something that has not yet been shown to be possible. 
A more realistic goal, at least in the short term, is the regeneration of single dental tissues, such as the pulp or dentine. Pulp cells for example are very easy to grow in culture and maintain a fibroblastic phenotype sufficient for endodontic applications. Indeed, clinical trials are in progress for autologous pulp cell therapy in endodontics. How practical and expensive such a therapy might turn out to be remains to be discovered, but as a first proof-of-principle that a cell therapy can work in a dental context, these trials are an important milestone.

Tooth conservation forms the cornerstone of the majority of dental treatment. Although levels of dental caries worldwide fell with the introduction of fluoride, recent surges largely attributed to high sugar diets have refocused interest on how caries lesions are treated more effectively. Removal of caries infected tooth mineral leaves a hole, a cavity that needs to be filled to protect the pulp and prevent recurrent infection. Calcium hydroxide remains widely used as pulp capping material although its relatively high failure rate has resulted in the introduction of more advances materials such as mineral trioxide (MTA) and Biodentine. These materials achieve a similar outcome to calcium hydroxide but have lower failure rate and provide some increased antibacterial activity. Never the less, the principle on which all these materials work is restoration of the cavity by filling with a non-biological material and does not result in any major regeneration of dentine. Dentine can however repair itself by regenerative processes that involve specific cell stimulation. Thus, in lesions where the pulp is exposed (deep caries) resident stem cells in pulp close to the exposed area are activated to differentiate into odontoblasts that produce a form of tertiary dentine (reparative dentine). In lesions that do not expose the pulp, odontoblasts are activated to produce reactionary dentine that results in a thickening of the dentine layer. The molecular and cellular mechanisms behind these processes are sufficiently well-understood to allow the development of novel biological therapies based on these natural processes, several of which are due to enter clinical trials soon.

Perhaps the biggest challenge faced by regenerative dentistry is in the treatment of periodontal disease. The periodontium is a very complex and poorly understood tissue and few successful attempts to apply regenerative approaches to "treat" periodontal disease have been reported. Cells that are grown in vitro from periodontal tissue that are described as "stem cells" are at best a highly heterogenous mixture and the nature and locations of resident stem cells in the PDL are not well-understood. What information is emerging shows that there are multiple stem cell populations playing different roles in the periodontium in vivo. From a clinical prespective, it is crucial to be able to regenerate periodontium in order to support the proper function of our dentition.

Harnessing biology to stimulate repair or regeneration is not without its challenges. Infection, inflammation, and immune rejection are all important considerations that need to better understood in the relevant context.

As already mentioned, the "holy grail" of regenerative dentistry remains the replacement of a tooth with a new tooth. Remarkable progress has been made in this area and bioengineering approaches that can produce cell implants that can develop into fully functional teeth (BioTeeth) in the mouth have been established. The biggest remaining hurdle is to identify ways of imparting tooth embryonic-like properties to adult cells that could be easily isolated from patients. Important clues to understanding how replacement teeth form naturally comes from evolutionary-based research that studies species that continuously replace their teeth such as snakes, reptiles, and fish.

Finally we should not forget that teeth exist in the context of the oral cavity that includes soft tissues and cells with important unique properties such as scar-free healing and an understanding of these is clearly part of what we can consider as regenerative dentistry.

The Regenerative Dentistry section of Frontiers in Dentistry aims to publish the highest quality research that impacts upon both our understanding of oral tissue regeneration and advance such understanding toward translation into clinical therapies. I personally hope to see more manuscripts that focus predominantly on in vivo investigation and make appropriate use of the very latest techniques and analyses. I have been able to assemble a great international team of Associate Editors with the expertise, experience and enthusiasm to help submission, review, and acceptance of the very best research in this area.

\section{AUTHOR CONTRIBUTIONS}

The author confirms being the sole contributor of this work and has approved it for publication.

Conflict of Interest: The author declares that the research was conducted in the absence of any commercial or financial relationships that could be construed as a potential conflict of interest.

Copyright (C) 2020 Sharpe. This is an open-access article distributed under the terms of the Creative Commons Attribution License (CC BY). The use, distribution or reproduction in other forums is permitted, provided the original author $(s)$ and the copyright owner(s) are credited and that the original publication in this journal is cited, in accordance with accepted academic practice. No use, distribution or reproduction is permitted which does not comply with these terms. 